\title{
Moderating effect of psychological resilience on the perceived social support and loneliness in the left-behind elderly in rural areas ${ }^{\dagger}$
}

Original article

\author{
Yue Liu, Lin Huang, Qin Liu, Gui-Zhen Qian, Huan Zou, Shan Zhang* \\ College of Nursing, Chengdu University of Traditional Chinese Medicine, Chengdu, Sichuan 610075, China
}

Received: 8 May 2021; Accepted: 7 June 2021; Published: 20 December 2021

\begin{abstract}
Objective: To investigate the loneliness and psychological resilience with the perceived social support of the left-behind elderly in rural areas and explore the moderating effect of psychological resilience on the understanding of social support and loneliness of the left-behind elderly in rural areas.

Methods: A cross-sectional study was conducted; 272 left-behind elderly people in 6 rural villages in the Sichuan Province were investigated by using the General Situation Questionnaire, Perceived Social Support Scale (PSSS), Connor-Davidson Resilience Scale (CD-RISC), and the University of California at Los Angeles Loneliness Scale (UCLA) with a stratified random cluster sampling method. Results: The loneliness score of the left-behind elderly in rural areas was $52.54 \pm 7.25$, the psychological resilience score was 78 (68.87), and the perceived social support score was 57 (51.62). The perceived social support and psychological resilience were negatively correlated with loneliness, while the perceived social support was positively correlated with psychological resilience. The moderating effect of psychological resilience on the perceived social support and loneliness of the left-behind elderly in rural areas was $-0.102(P<0.001)$.

Conclusions: Higher level resilience is of positive significance to alleviate the negative effect of social support on loneliness. Improving the resilience of the left-behind elderly in rural areas is a beneficial exploration to promote their mental health.
\end{abstract}

Keywords: loneliness $\bullet$ moderating effect $\bullet$ perceived social support $\bullet$ psychological resilience $\bullet$ the left-behind elderly

(c) Shanxi Medical Periodical Press.

\section{Introduction}

The research report on left-behind elderly in rural China (2018) pointed out that the number of left-behind elderly in rural China had reached 16 million, accounting for
$47.08 \%$ of the "three left-behind" populations, and was the main part of the "three left-behind" groups. Due to their children's migrant work and their physiological function degeneration, the left-behind elderly are more likely to have negative psychology, while loneliness is the

†This project was supported by the Primary Health Development Research Center of Sichuan Province (No. SWFZ19-Q-03).

How to cite this article: Liu Y, Huang L, Liu Q, Qian GZ, Zou H, Zhang S. Moderating effect of psychological resilience on the perceived social support and loneliness in the left-behind elderly in rural areas. Front Nurs. 2021;4:357-364. 
most important psychological problem. Loneliness is a negative emotional state that occurs when individuals feel socially isolated or experience negative social interactions. ${ }^{1}$ Loneliness in old age is significantly related to cognitive function, cardiovascular disease, anxiety, depression, and even suicidal ideation, and higher levels of loneliness significantly affect the quality of life of the elderly. ${ }^{2}$ Perceived social support refers to the social support of individual subjective experience, which is negatively correlated with loneliness, as well as is the main external factor for the analysis of loneliness in previous studies. ${ }^{3,4}$ Psychological resilience is an individual's positive ability to adapt to adverse environmental conditions such as pressure, trauma, frustration, and threat. It is also an internal protective factor to maintain an individual's mental health. It plays an important role in whether an individual can effectively cope with negative stimuli to improve the adaptability to the environment. ${ }^{5}$

Research showed that the perceived social support was a positive predictor of psychological resilience. ${ }^{6-8}$ Therefore, we speculate that the psychological resilience of the left-behind elderly may be related to their loneliness, and the perceived social support affects the development of loneliness of the left-behind elderly. But the exact relationship between perceived social support, psychological resilience, and loneliness remains unclear.

Based on the above statement, from the perspective of positive psychology, this study firstly investigated the loneliness and psychological resilience with the perceived social support of the left-behind elderly in rural areas, secondly analyzed internal relations among the three parameters, and thirdly verified the moderating effect of psychological resilience between perceived social support and loneliness, so as to reduce the loneliness of the left-behind elderly through the internal factors working together with external factors, moreover, to provide theoretical basis of formulating intervention measures to alleviate the loneliness of the rural left-behind elderly.

\section{Methods}

\subsection{Participants}

The stratified random cluster sampling method was adopted to select 272 rural left-behind elderly people from 6 villages including 2 villages of each town in central Sichuan, south Sichuan, and north Sichuan. Inclusion criteria are as follows: (1) age $\geq 60$ years old; (2) willing to participate in the survey and obtaining informed consent; and (3) meeting a criterion of rural left-behind old people: The elderly stayed in the village whose children all leave the county scope to go out for work or business for more than half a year with no supporter left behind with them or the supporter having no ability to support.
(1) Elderly with severe mental disorders and cognitive impairment and (2) elderly with physical diseases that cannot cooperate are excluded from the study.

\subsection{Instrument}

\subsubsection{The general information questionnaire}

The general information questionnaire of the elderly includes age, gender, marital status, current living status, educational level, whether they engage in recreational activities at ordinary times, their children's migrant work time, their children's contact frequency, and their attitude toward their children's migrant work.

\subsubsection{The Perceived Social Support Scale}

The Perceived Social Support Scale (PSSS) was made by Zimet et $\mathrm{al}^{9}$ and translated into Chinese by Jiang Qianjin, ${ }^{10}$ which consists of 12 items and three dimensions including family support, friend support, and other support, emphasizing the degree of social support felt by the individual. The seven-item Likert scale is used with scores ranging from strongly disagree to strongly agree, with a score of 1-7 for each item and a total score of 12-84. The higher the scores are, the better the degree of understanding of social support would be perceived. The Cronbach's $\alpha$ coefficient of the scale was $0.93 .{ }^{11}$

\subsubsection{Connor-Davidson Resilience Scale}

The Connor-Davidson Resilience Scale (CD-RISC) was developed by Connor et al. ${ }^{12}$, which was designed to measure the ability to cope with adversity. The Chinese version of the CD-RISC developed by Yu et al. ${ }^{13}$ includes 25 items from the original scale, covering three dimensions of tenacity, strength, and optimism, using the fiveitem Likert scale whose total score ranges from 0 to 100 . The higher the scores are, the higher the level of psychological resilience would be obtained. This scale had good reliability and validity in the Chinese elderly population. ${ }^{14}$

\subsubsection{The University of California at Los Angeles Loneliness Scale}

The University of California at Los Angeles Loneliness Scale (UCLA) was developed by Russell et al. ${ }^{15}$ including 9 items of "non-lonely" reverse scoring items and 11 items of "loneliness" forward scoring items. Each item was scored at 4 levels (never, rarely, sometimes, and all the time), with a total score of $20-80$ points. The higher the scores are, the more serious the loneliness would be felt. The Cronbach's $\alpha$ coefficient was proved to be 0.883 in the elderly population. ${ }^{3}$ 


\subsection{Data collection}

In July 2020, 272 rural left-behind elderly people in Sichuan Province were investigated by an on-site questionnaire survey. Unified training investigators explained the purpose of the research to the elderly and obtained oral informed consent; then, they issued questionnaires and instructed the elderly to fill the questionnaires in front of them. For the elderly who could not read or had a low level of education, the investigator objectively reported the items of the scale. The questionnaires were anonymous, and double-entry was performed with Epidata, version 3.0 (The EpiData Association, Odense Denmark, 2003). In this study, a total of 272 questionnaires were sent out, and those with incomplete questionnaire information were excluded. Two hundred sixty valid questionnaires were collected with an effective recovery rate of $95.6 \%$.

\subsection{Statistical analysis}

SPSS, version 25.0, and AMOS, version 24.0 (IBM Corporation, Armonk, New York, United States), were used for statistical analysis. The measurement data of normal distribution were described as mean \pm standard deviation, and the measurement data of non-normal distribution were described as median (quartile range). Due to the non-normal distribution of UCLA scores in each dimension of general data, the Wilcoxon test and Kruskal-Wallis $H$ test were used for monofactor analysis. Spearman correlation analysis was used to analyze the correlation between perceived social support, psychological resilience, and loneliness of left-behind elderly. Structural equation models (SEMs) were used to examine the moderating effect of psychological resilience on perceived social support and loneliness. A value of $P<0.050$ was considered as statistically significant.

\section{Results}

\subsection{Comparison of UCLA scores for the left- behind elderly in rural areas $(n=260)$}

Among the 260 participants, there were statistically significant differences in their UCLA scores among different ages, marital status, current living status, whether they had recreational activities or not, and the contact frequency of their children. However, there was no statistically significant difference in UCLA scores among different genders, educational level, children's migrant work years, and attitudes toward their children's migrant work, as given in Table 1.

\subsection{The correlation between loneliness, psychological resilience, and the perceived social support among the left-behind elderly in rural areas}

In this study, the left-behind elderly in rural areas got 57 (51.62) points in the PSSS for each dimension with a score of 20 (17.22) points for friend support, 20 (17.23) points for other support, and 17 (15.19) points for family support, along with the scoring rates of $70.61 \%$, $71.21 \%$, and $58.75 \%$, respectively. The scores in the CD-RISC were 78 points (68.87), among which the scores of each dimension were 44 (38.48), 24 (21.27), and 10 (8.12) points, with the scoring rates of $64.83 \%$, $60.65 \%$, and $49.45 \%$, respectively, for friend support, other support, and family support. UCLA scores were $52.54 \pm 7.25$. The correlation analysis results showed that there was a positive correlation between perceived social support and psychological resilience $(r=0.190$, $P<0.010$ ), while the correlation between perceived social support and loneliness was negative $(r=-0.542$, $P<0.010)$. Meanwhile, psychological resilience was negatively correlated with loneliness $(r=-0.348$, $P<0.010$ ), as shown in Table 2 .

\subsection{SEM test on the moderating effect of psychological resilience on the perceived social support and loneliness of left-behind elderly in rural areas}

To make up for the deficiency of the moderating effect of hierarchical regression tests, ${ }^{16}$ this study based on the calculation method of ping's regulating variable conducted an SEM to examine the moderating effect of psychological resilience on the perceived social support and loneliness in rural left-behind elderly. ${ }^{17}$ The first step was to estimate the factor load and residuals of the main effect in the model. In the second step, the estimated results were used to calculate the factor load and residuals of the interaction term, which were taken as the constants in the model containing the interaction term. If the interaction effect was significant, then the interaction effect would exist. The results showed that the fitting index of the model had the following values: $\chi^{2}$ dff value (2.318), RMSEA value (0.071), AGFI value (0.934), CFI value (0.975), and TLI value (0.957), all of which were within the acceptable range, indicating that the model fitted well; the factor load of the interaction item on loneliness was $-0.102(P<0.001)$, indicating that psychological resilience had a moderating effect on the perceived social support and loneliness. The specific model is shown in Figure 1. The moderating effect is shown in Figure 2. 


\begin{tabular}{|c|c|c|c|c|}
\hline Variable & Cases & Loneliness scores & Mean rank & $\mathrm{Z} / \chi^{2}$ value \\
\hline Age (years) & & & & $6.643^{*}$ \\
\hline $60-69$ & 115 & $52(46,57)$ & 124.38 & \\
\hline $70-79$ & 81 & $52(47,56)$ & 122.62 & \\
\hline$\geq 80$ & 64 & $55(50,60)$ & 151.47 & \\
\hline Gender & & & & -1.133 \\
\hline Female & 134 & $52(48,57.25)$ & 135.62 & \\
\hline Male & 126 & $52(48,56.5)$ & 125.06 & \\
\hline Marital status & & & & $8.140^{\star}$ \\
\hline Married & 160 & $52(46,56.75)$ & 120.00 & \\
\hline Widowed & 76 & $54(51,60)$ & 147.74 & \\
\hline Unmarried & 24 & $54(49.25,57)$ & 145.92 & \\
\hline Living status & & & & $10.289^{\star \star *}$ \\
\hline With the spouse & 140 & $51(46,56)$ & 116.73 & \\
\hline Alone & 74 & $54(50,60.50)$ & 148.2 & \\
\hline With others & 46 & $54(49,57.25)$ & 143.93 & \\
\hline Education & & & & 4.589 \\
\hline Illiteracy or primary school & 165 & $53(48,58)$ & 135.09 & \\
\hline Junior high school & 49 & $54(48,58.5)$ & 135.23 & \\
\hline Senior high school & 46 & $52(44.75,53)$ & 108.98 & \\
\hline Recreational activities & & & & $-3.752^{\star \star}$ \\
\hline Yes & 128 & $52(44.5,55.75)$ & 112.75 & \\
\hline No & 132 & $54(49,60)$ & 147.71 & \\
\hline Children's migrant work years & & & & 4.383 \\
\hline$\geq 5$ years & 204 & $52(47,57)$ & 126.06 & \\
\hline $3-5$ years & 35 & $54(49,60)$ & 154.73 & \\
\hline $1-3$ years & 21 & $53(49.5,60)$ & 133.26 & \\
\hline Children's contact frequency & & & & $7.253^{*}$ \\
\hline Never & 48 & $54(49.25,62)$ & 155.21 & \\
\hline 1-2 times per week & 120 & $52(48,47)$ & 129.15 & \\
\hline 3-5 times per week & 92 & $52(46,55)$ & 119.37 & \\
\hline Attitudes toward their children's migrant work & & & & 4.586 \\
\hline Support & 212 & $52(48,57)$ & 127.62 & \\
\hline It does not matter & 40 & $54(49,57)$ & 134.95 & \\
\hline Against & 8 & $57(54,60)$ & 184.50 & \\
\hline
\end{tabular}

Note: ${ }^{*} P<0.050 ;{ }^{*} P<0.010$.

UCLA, University of California at Los Angeles Loneliness Scale.

Table 1. Comparison of UCLA scores for the left-behind elderly in rural areas $(n=260)$.

\begin{tabular}{lccc}
\hline Perceived social support & & $\begin{array}{c}\text { Psychological } \\
\text { resilience }\end{array}$ & Loneliness \\
\hline \hline Perceived social support & 1.000 & & \\
Psychological resilience & $0.190^{\star}$ & 1.000 & \\
Loneliness & $-0.542^{\star}$ & $-0.348^{\star}$ & 1.000 \\
\hline
\end{tabular}

Note: ${ }^{*} P<0.010$.

Table 2. Correlation between perceived social support, psychological resilience, and loneliness among the left-behind elderly in rural areas ( $R$-value).

\section{Discussion}

\subsection{The loneliness of the left-behind elderly in rural areas}

In this study, the loneliness score of the rural left-behind elderly was $52.54 \pm 7.25$, which was basically the same as the survey of Yang Qingsong et al. and Zhang Chunlin et al., while significantly higher than the norm of loneliness for the elderly $(31.5 \pm 6.9)$, indicating that 


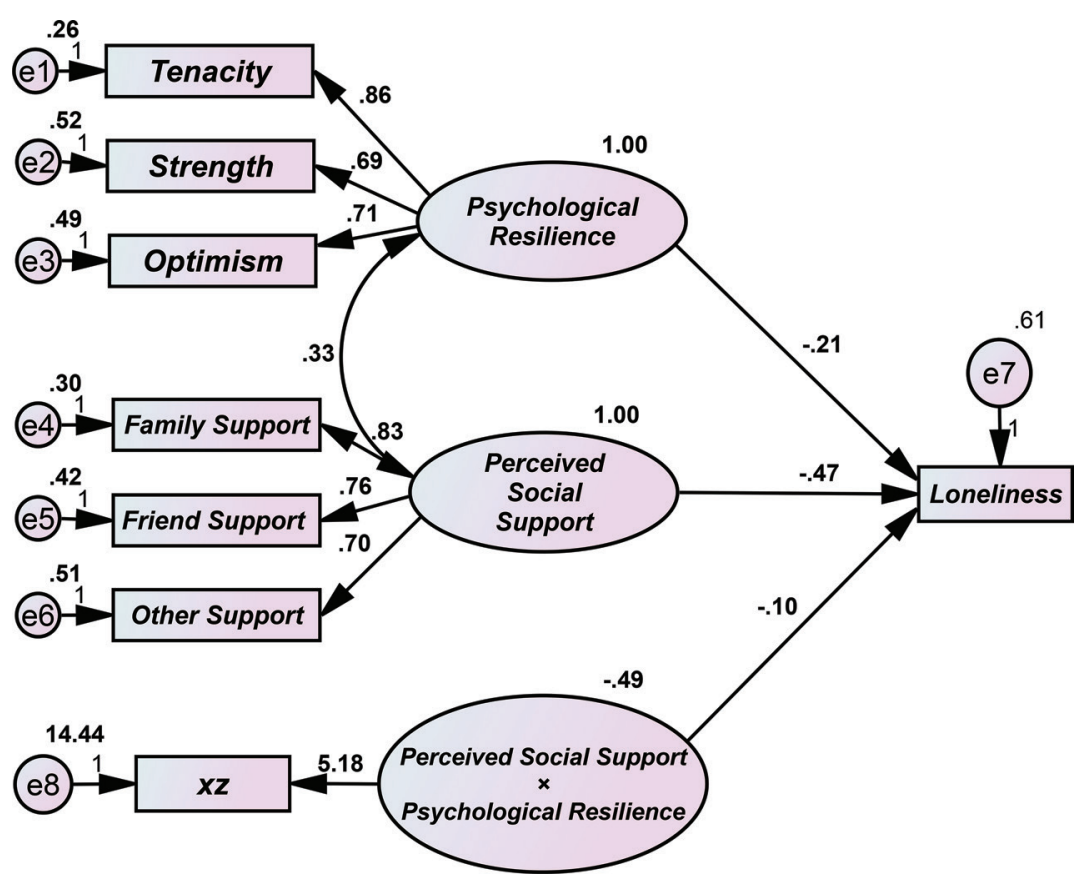

Figure 1. The moderating effect model of psychological resilience on perceived social support and loneliness (standardization). Note: $x z$ is the product of addition and then normalization of the dimensions of social support and resilience.

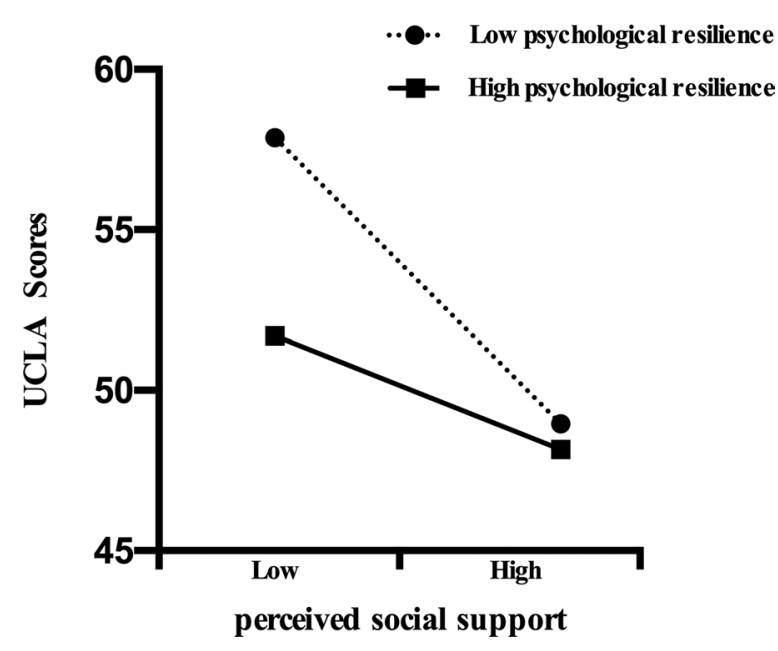

Figure 2. The moderating effect of psychological resilience on the perceived social support and loneliness among the left-behind elderly in rural areas. UCLA, University of California at Los Angeles Loneliness Scale.

the left-behind elderly in rural areas has a strong sense of loneliness, which needs to be alleviated. The monofactor analysis showed that there was no significant difference in loneliness among the left-behind elderly with different educational levels, which was inconsistent with the past research that may be due to the leftbehind elderly in rural areas being not generally highly educated with no significant differences among their psychological resources. In addition, the left-behind elderly who are widowed, live alone, deprived of recreational activities, and have a low frequency of contact with their children had a higher level of loneliness, ${ }^{1}$ which may be related to the lack of necessary emotional support and attention to the left-behind elderly and the unstable participation in social activities, pointing out that more attention should be paid to these elderly people to help them expand their social circle and strengthen their mental health. In this study, the psychological resilience of the rural left-behind elderly was lower than that of the elderly in the community, which may be because of the low level of economics, educations, living conditions, etc. of the left-behind elderly. The optimistic dimension served a minimum suggesting that the rural clerks should strengthen psychological counseling for the old and pay close attention to their economic income; then, they should prepare psychological construction of knowledge propaganda and lectures and group psychological counseling, so that the elderly have an optimistic attitude to face the pressure. In this study, the left-behind elderly in rural areas scored lower on perceived social support, which was consistent with previous studies, indicating that rural left-behind old people have a low level of understanding of social support, who should be helped to establish a more social network. The family dimension score rate was the lowest in the PSSS, suggesting that children should contact and care about their parents regularly, so that the elderly can feel more affection and warmth from the family. 


\subsection{Relationship among loneliness, perceived social support, and psychological resilience}

The results of this study showed that while the higher the social support was perceived and the higher the psychological resilience of the left-behind elderly was obtained, the lower the loneliness would be obtained and vice versa. This was consistent with previous studies, showing that the more social support individuals perceive, the more confidence in interpersonal communication would be enhanced, more social interactions would be established, so that reducing their loneliness. This correlation had been reported in children and adolescents until now but has been confirmed in the rural leftbehind elderly population in this study. In addition, the results were in accordance with the main effect model of social support, ${ }^{18}$ in which social support was used to overcome stress and promote mental health. Thus, to improve understanding of social support is an important approach to alleviate the left-behind elderly loneliness, children flowing out should strengthen emotional exchange and communication with the left-behind parents, at the same time, the rural clerks should advocate the neighborhood assistance and interaction among the elderly, and regularly provide psychological care for old people to enhance their recognition from practical social support. Meanwhile, psychological resilience also had a negative predictive effect on loneliness. Psychological resilience is considered as an important protective mechanism for individuals in the face of setbacks. ${ }^{19}$ Individuals with high psychological resilience can make more use of their internal and external resources to cope with difficulties, so as to achieve good adaptation and buffer psychological problems such as loneliness. ${ }^{20}$ Therefore, the results also suggested that improving the psychological resilience and positive adaptability of leftbehind elderly may be one of the effective measures to alleviate their loneliness.

\subsection{Psychological resilience adjusts the relationship between perceived social support and loneliness for the rural left-behind elderly}

This study showed that the old man's psychological resilience played moderating effects between perceived social support and loneliness, in other words, psychological resilience can buffer the influence of low level perceived social support on loneliness, probably because higher psychological resilience got by the leftbehind elderly, can strengthen their faith to overcome low level perceived social support, and improve the negative mood, instead, lower psychological resilience will magnify the pressure from low level perceived social support as well as negative emotions. The results of this study were similar to those of the model of psychological resilience proposed by Kumpfer, ${ }^{21}$ that is, psychological resilience as a protective factor can offset the negative consequences of individuals in a dangerous environment and help individuals actively cope with and regulate stressors, which may also partially reveal the boundary conditions for the impact of perceived social support on loneliness. Therefore, a higher level of psychological resilience is also of positive significance to alleviate the loneliness of the left-behind elderly. It is found that the perceived social support is closely related to the actual degree of support obtained by individuals, which is sometimes difficult to change due to their social objectivity. Psychological resilience is a dynamic concept that can be changed and learned throughout a person's life. ${ }^{6}$ Therefore, the rural clerks should strengthen the screening of left-behind elderly loneliness levels during the family visit and develop intervention plans with reference to the theory of psychological resilience; meanwhile, they should regularly carry out psychological resilience promotion training to make up for the influence of insufficient perceived social support on the loneliness of the left-behind elderly and promote their positive psychological level, so as to reduce their loneliness and improve their quality of life.

\section{Conclusions}

This study used the SEM research method to explore the relationship among perceived social support, psychological resilience, and loneliness of rural left-behind elderly; on the one hand, it solved the statistical bias from the interactions of multiple factors, which was the improvement compared with previous research by the hierarchical regression statistical method. On the other hand, this study explored the relationship among perceived social support, psychological resilience, and loneliness not only to find and analyze problems but also to further propose solutions to the problems found, which provided a theoretical basis for improving the mental health level of the rural left-behind elderly. Although the effective sample size of this study was only 260 cases, it met the requirement of the SEM model with a sample size $>150$ cases, and the stratified random cluster sampling method is adopted to ensure the reliability of the research data. It was a cross-sectional study, only reflecting the correlation of the three parameters but being unable to prove a causal relationship. The next step is proposed to use the longitudinal study to explore causal relations among the three and detect more protective factors and paths through psychological resilience to adjust the influence of perceived social support on loneliness, formulating targeted intervention strategy, so as to promote the mental health level of the 
rural left-behind elderly by the combination of internal factors and external factors.

\section{Ethical approval}

Ethical issues are not involved in this article. The rural left-behind elderly who voluntarily participated were informed about the purpose of the study, and this study would not have any negative impact on them.

\section{Conflicts of interest}

All authors have nothing to disclose.

\section{References}

1. Wang G, Hu M, Xiao SY, Zhou L. Loneliness and depression among rural empty-nest elderly adults in Liuyang, China: a cross-sectional study. BMJ Open. 2017;7:e16091.

2. Emerson KG, Jayawardhana J. Risk factors for loneliness in elderly adults. J Am Geriatr Soc. 2016;64:886-887.

3. Wu J, Li HQ, Xu S. Loneliness and the impact of understanding social support on mental health of the elderly. Chin J Health Psychol. 2017;25:18371840 (in Chinese).

4. Su SW. A study on the relationship between social support, loneliness and health of the elderly under different pension modes. Guangzhou: Southern Medical University; 2019 (in Chinese).

5. Peterson BS, Wang Z, Horga G, et al. Discriminating risk and resilience endophenotypes from lifetime illness effects in familial major depressive disorder. JAMA Psychiatry. 2014;71:136-148.

6. van Kessel G. The ability of older people to overcome adversity: a review of the resilience concept. Geriatr Nurs. 2013;34:122-127.

7. Wu M, Yang Y, Zhang D, et al.. Association between social support and health-related quality of life among Chinese rural elders in nursing homes: the mediating role of resilience. Qual Life Res. 2018;27:783-792.

8. Janssen BM, Van Regenmortel T, Abma TA. Identifying sources of strength: resilience from the perspective of older people receiving long-term community care. Eur J Ageing. 2011;8:145-156.

9. Zimet GD, Powell SS, Farley GK, Werkman S, Berkoff KA. Psychometric characteristics of the multidimensional scale of perceived social support. J Pers Assess. 1990;55:610-617.

10. Wang XD, Wang XL, Ma H. Handbook of Mental Health Rating Scale. Beijing: Chinese Mental Health Journal; 1999 (in Chinese).
11. Zhang BL. A Study on the Relationship Between Resilience, Perception of Social Support and Depression in Younger Aged Adults. Tianjin: Nankai University; 2011 (in Chinese).

12. Connor KM, Davidson JR. Development of a new resilience scale: the Connor-Davidson Resilience Scale (CD-RISC). Depress Anxiety. 2003;18:76-82.

13. Yu X, Zhang J, Yu X N. Factor analysis and psychometric evaluation of the Connor-Davidson Resilience Scale (CD-RISC) with Chinese people. Soc Behav Pers Int J. 2007;35:19-30.

14. Zhang J, Zhang JP, Wang AN, Su P. Reliability and validity of the Chinese version of resilience scale in community empty-nesters. Chin J Gerontol. 2017;37:3611-3613 (in Chinese).

15. RussellD, Peplau LA, Ferguson ML. Developing a measure of loneliness. J Pers Assess. 1978;42:290-294.

16. Cortina JM, Chen G, Dunlap WP. Testing interaction effects in LISREL: examination and illustration of available procedures. Organ Res Methods. 2001;4:324-360.

17. Ping RA Jr. A parsimonious estimating technique for interaction and quadratic latent variables. Multivar Behav Res. 1995;32:336-347.

18. Cohen S, Wills TA. Stress, social support, and the buffering hypothesis. PsycholBull. 1985;98:310-357.

19. Bonanno GA. Loss, trauma, and human resilience: have we underestimated the human capacity to thrive after extremely aversive events? Am Psychol. 2004;59:20-28.

20. Gerino E, Rolle L, Sechi C, Brustia P. Loneliness, resilience, mental health, and quality of life in old age: a structural equation model. Front Psychol. 2017;8:2003.

21. Kumpfer KL. Factors and Processes Contributing to Resilience: The Resilience Framework. New York: Kluwer Academic/Plenum Publishers; 2002. 